\title{
Stable boundary layers in a diffusion problem with nonlinear reaction at the boundary
}

\author{
José M. Arrieta * \\ Neus Cónsul ${ }^{\dagger}$ \\ Aníbal Rodríguez-Bernal *
}

\section{Introduction}

In this paper we consider the evolution problem with a nonlinear reaction acting on the boundary

$$
\left\{\begin{array}{rlrl}
u_{t}-\varepsilon \Delta u & =0, & & \text { in } \Omega, t>0 \\
\varepsilon \frac{\partial u}{\partial n}+g(x, u) & =0, & & \text { on } \Gamma, t>0 \\
u(\cdot, 0) & =\psi(\cdot) \in H^{1}(\Omega) . &
\end{array}\right.
$$

and study the existence and behavior as the parameter $\varepsilon$ goes to zero of non constant stable equilibria. Such type of solutions are denoted patterns. In our setting, $\Omega \subset \mathbb{R}^{2}$ is a bounded domain with a $C^{1}$ and connected boundary $\Gamma$ and $\frac{\partial}{\partial n}$ denotes the outward normal derivative. The nonlinearity is given by

$$
g(x, u)=u(1-u)(c(x)-u), \quad x \in \Gamma, u \in \mathbb{R},
$$

where $c \in \mathcal{C}^{1}(\Gamma)$ satisfies $0<c(x)<1$ for all $x \in \Gamma$, and the transversality condition

$$
\frac{\partial c}{\partial \tau}(z) \neq 0
$$

for all $z \in \Gamma$ with $c(z)=1 / 2$, where $\tau$ is the unit tangent vector at the boundary. Let us note that this condition implies that there exist only a finite and even number of points $x_{i}, i=1, \ldots, 2 N$ satisfying $c\left(x_{i}\right)=1 / 2$.

* partially supported by BFM2000-0798

${ }^{\dagger}$ partially supported by CICYT PB98-0932-C02-01 
In [4] we showed the existence of patterns, $u_{\varepsilon}$, for $\varepsilon$ small enough which are close to a limit profile $u_{0}$. These equilibria satisfy

$$
\left\{\begin{aligned}
-\varepsilon \Delta u & =0, & & \text { in } \Omega, \\
\varepsilon \frac{\partial u}{\partial n}+g(x, u) & =0, & & \text { on } \Gamma,
\end{aligned}\right.
$$

and they present layers at the boundary located at the points $x_{i}$, that we will denote by transition points. These patterns are obtained as global minimizers of the appropriate energy functional related to problem (1.1). Moreover the trace of $u_{0}$ at the boundary is a characteristic function with values 0 where $c(x)>1 / 2$ and 1 where $c(x)<1 / 2$.

Observe that there are some results on the literature about the existence or non existence of patterns for some other similar problems. If the nonlinearity at the boundary does not have a spatial dependence, that is, $g(x, u)=g(u)$ and $\Omega$ is a ball in $\mathbb{R}^{N}, N \geq 2$, it is known that there are no patterns for any $\varepsilon>0$, that is, only constant solutions can be stable equilibria, see [5]. Also, it is known that for problem (1.1) with $\varepsilon=1$, the formation of patterns can be achieved with a typical bistable cubic nonlinearity with no spatial dependence, $g(u)=u-u^{3}$, when the domain looses its convexity. This is the case of the so called dumbbell domains which consist of two disjoint fixed domains joined by a thin channel, see $[6,7]$. Patterns also appear when the boundary of the domain $\Omega$ is disconnected, like a domain with holes, see [5]. Therefore here we show that spacedependent boundary flux provides a mechanism for pattern formation even on convex domain with connected boundary.

These results have been obtained very much in the spirit of the famous results presented in $[9,10]$ which assert the non existence of patterns in convex domains for some reaction diffusion equations with Neumann boundary conditions.

In this direction, the one dimensional problem given by

$$
\begin{cases}u_{t}=\varepsilon^{2} u_{x x}+f(x, u), & 0<x<1, t>0, \\ u_{x}(0, t)=u_{x}(1, t)=0, & t>0, \\ u(x, 0)=\phi(x), & 0 \leq x \leq 1 .\end{cases}
$$

with $\varepsilon \in \mathbb{R}^{+}$and $f(x, u)=u(1-u)(u-a(x))$ was considered in [1]. The function $a(\cdot) \in \mathcal{C}^{1}([0,1])$ satisfies $0<a(x)<1$, for all $x \in[0,1]$ and a transversality condition similar to (1.2). For $\varepsilon$ small, a complete classification of all stable equilibrium solutions of problem (1.4) was given in that paper. In [11] some similar results are obtained allowing the function $a(\cdot)$ being discontinuous.

Recently in [8], similar results have been proven for higher dimensional problems of the form

$$
\begin{cases}u_{t}=\varepsilon \nabla \cdot(d(x) \nabla u)+\left(1-u^{2}\right)(u-a(x)), & (t, x) \in \mathbb{R}^{+} \times \Omega, \\ \frac{\partial u}{\partial n}=0, & \partial \Omega, t>0 \\ u(x, 0)=\phi(x) . & \end{cases}
$$


where $\Omega \subset \mathbb{R}^{N}, N \geq 2$. Here the function $a(\cdot) \in \mathcal{C}^{0, \nu}(\Omega)$ and satisfies $-1<a(x)<1$ and vanishes on some hypersurfaces.

There are some obvious differences between problems (1.4) and (1.1) due to the dimension of $\Omega$ and the presence of a nonlinear Neumann boundary condition. In certain sense, problem (1.3) is a $1+\frac{1}{2}$ dimensional problem. Hence, most of the basic tools for the one dimensional problem, e.g. sub and super solutions, the underlying second order boundary value problem, etc., are no longer available.

In this paper we study the convergence of the patterns $u_{\varepsilon}$ to $u_{0}$ in different norms, including $C_{\text {loc }}^{\infty}(\Omega)$ and in $L^{\infty}\left(\bar{\Omega} \backslash\left\{x_{1}, \ldots, x_{2 N}\right\}\right)$. That is, we show that the patterns converge, as $\varepsilon \rightarrow 0$, strongly in the sup norm outside small neighborhoods of the transition points. Notice that the limit profile has jumps at these points and therefore it is not possible to obtain an $L^{\infty}$ convergence in the whole domain. To obtain this result we need to perform a careful analysis of the behavior of the patterns near the boundary. This is done in Section 3.

Once the patterns have been obtained it is important to study their stability regions as the parameter goes to zero. We will show the existence of $\varepsilon$-independent nested regions, $R_{n}$, around the limiting profile $u_{0}$ with the property that if an initial condition is given in $R_{n}$ and if $\varepsilon$ is small enough the solution of (1.1) with this initial condition will remain in $R_{n-1}$ for all time. The fact that these regions are independent of $\varepsilon$ means that the profile of the patterns is persistent as $\varepsilon$ approaches zero. This is done in Section 4.

We also include in Section 2 a brief summary of the results obtained previously in [4], which are needed for the rest of the paper.

There still remains many interesting questions for this problem, like for instance, the asymptotic stability and uniqueness of the patterns, the monotonicity of the layers at the boundary and, in general, the shape of the layers.

\section{Existence of non-constant stable equilibria}

In this section we recall and summarize earlier results announced by the authors in [4]. These results state the existence of nontrivial stable equilibria for (1.1) and also some preliminary description of their shape as $\varepsilon \rightarrow 0$.

Associated to problem (1.1) we have the energy functional $J_{\varepsilon}: H^{1}(\Omega) \rightarrow \mathbb{R}$ defined by

$$
J_{\varepsilon}(u)=\frac{\varepsilon}{2} \int_{\Omega}|\nabla u|^{2}+\int_{\Gamma} G(x, u) d x
$$

where $G(x, u)=\int_{0}^{u} g(x, s) d s$. This energy acts as a Lyapunov function for the solutions of (1.1), since it strictly decreases in time except at equilibria. 
Note that natural candidates for stable equilibrium solutions are absolute minimizers of the energy functional (2.1), whose existence is guaranteed by the direct method of calculus of variations. Moreover, by maximum principles, these equilibria take values between 0 and 1 .

To prove that global minimizers are non constant we actually show that, as $\varepsilon \rightarrow 0$, they are close to some non constant profile, $u_{0}(x)$, which is obtained as follows. Observe first that for fixed $x \in \Gamma$, the function $s \in \mathbb{R} \rightarrow G(x, s)$ has an absolute minimum at $s=0$ if $c(x)>1 / 2$ and at $s=1$ if $c(x)<1 / 2$. This implies that for any function $u(\cdot)$ defined on $\Gamma$ we have $\int_{\Gamma} G(x, u(x)) d x \geq \int_{\Gamma} G(x, \chi(x)) d x=m_{0}$, where $\chi(x)$ denotes characteristic function on the boundary given by

$$
\chi(x)= \begin{cases}1, & x \in \Gamma, \text { with } c(x)<1 / 2, \\ 0, & x \in \Gamma, \text { with } c(x)>1 / 2 .\end{cases}
$$

Since this function minimizes the boundary term in the energy (2.1), it is natural to guess that a global minimizer of the full energy would be obtained by considering the harmonic extension to $\Omega$ of $\chi, u_{0}$ which satisfies

$$
\begin{cases}\Delta u_{0}=0, & \text { in } \Omega, \\ u_{0}(x)=\chi(x), & \text { on } \Gamma .\end{cases}
$$

However, $\chi \notin H^{1 / 2}(\Gamma)$ and therefore $u_{0} \notin H^{1}(\Omega)$, so it is not actually a minimizer of the energy. Moreover, it can be shown that $0 \leq u_{0} \leq 1, u_{0} \in H^{s}(\Omega)$ for any $s<1$, $u_{0} \in C^{\infty}(\Omega)$ and in fact $u_{0} \in C^{0}\left(\bar{\Omega} \backslash x_{1}, \ldots, x_{2 N}\right)$.

To prove that global minimizers approach somehow the profile $u_{0}$, as $\varepsilon \rightarrow 0$, we proceed as follows. By mollification on the boundary we construct functions $\chi_{\varepsilon} \in C^{1}(\Gamma)$ with $0 \leq \chi_{\varepsilon} \leq 1$ such that $\chi_{\varepsilon}=\chi$ in $\Gamma_{\varepsilon}$, where $\Gamma_{\varepsilon}=\Gamma \backslash \cup_{i=1}^{2 N} B\left(x_{i}, \varepsilon\right)$. Using Fourier transform we show that the harmonic extension to $\Omega$ of $\chi_{\varepsilon}$, that we denote by $v_{\varepsilon}$, satisfies $\left\|\nabla v_{\varepsilon}\right\|_{L^{2}(\Omega)} \leq C|\log (\varepsilon)|$ and therefore $J_{\varepsilon}\left(v_{\varepsilon}\right) \leq m_{0}+C_{0} \varepsilon|\log (\varepsilon)|$ for some constant $C_{0}$ independent of $\varepsilon$.

In particular this implies that global minimizers lie in the set $K_{\varepsilon}=\left\{u \in H^{1}(\Omega) ; J_{\varepsilon}(u)<\right.$ $\left.m_{0}+C_{0} \varepsilon|\log (\varepsilon)|\right\}$ which is open, nonempty and positively invariant for (1.1). Moreover absolute minimizers satisfy that

$$
\int_{\Omega}\left|\nabla u_{\varepsilon}\right|^{2} \leq C|\log (\varepsilon)|
$$

for some constant $C$ independent of $\varepsilon$.

From here and the transversality condition on the coefficient $c(x)$ we are able to show that

$$
\left\|u_{\varepsilon}-\chi\right\|_{L^{2}(\Gamma)}+\left\|u_{\varepsilon}-u_{0}\right\|_{L^{2}(\Omega)} \leq C(\varepsilon|\log (\varepsilon)|)^{\frac{1}{4}}
$$

This implies the convergence in $L^{p}(\Omega)$ and $L^{p}(\Gamma)$ for any $1 \leq p<\infty$. 


\section{$3 \quad L^{\infty}$-convergence of minimizers outside transition points}

In the previous section we have obtained the convergence of minimizers in $L^{p}(\Omega)$ and in $L^{p}(\Gamma)$ for any $1 \leq p<\infty$. Since the limit function $u_{0}$ is discontinuous at the transition points, it is not possible to obtain convergence in $L^{\infty}(\Omega)$ or $L^{\infty}(\Gamma)$. Nevertheless, we will show in this section that if we avoid the transition points, the minimizers will converge in the norm of the supremum. To obtain this result we will need to perform a careful analysis of the behavior of the function $u_{\varepsilon}$ near the boundary $\Gamma$ and outside the transition points $\left\{x_{1}, \ldots, x_{2 N}\right\}$. As a matter of fact we can show the following result

Proposition 3.1 The convergence of $u_{\varepsilon}$ to $u_{0}$ holds in $C_{l o c}^{\infty}(\Omega)$ and in $L_{l o c}^{\infty}\left(\bar{\Omega} \backslash\left\{x_{1}, \cdots, x_{2 N}\right\}\right)$.

Before proving the result, let us state and prove the following technical lemma that will be needed below.

Lemma 3.2 If $\phi \in C^{\infty}(\bar{\Omega})$ and $u$ is a harmonic function which is regular on supp $(\phi)$ we have

$$
\int_{\Omega}|\nabla(u \phi)|^{2}=\int_{\Omega} u^{2}|\nabla \phi|^{2}+\int_{\Gamma} \phi^{2} u \frac{\partial u}{\partial n} .
$$

Proof of Lemma 3.2. Integrating by parts one obtains

$$
\begin{aligned}
\int_{\Omega}|\nabla(u \phi)|^{2} & =\int_{\Gamma} \frac{\partial(u \phi)}{\partial n} u \phi-\int_{\Omega} \Delta(u \phi) u \phi \\
& =\int_{\Gamma} \frac{\partial u}{\partial n} u \phi^{2}+\int_{\Gamma} \frac{\partial \phi}{\partial n} u^{2} \phi-\int_{\Omega} 2 \nabla u \nabla \phi u \phi-\int_{\Omega} u^{2} \Delta \phi \phi \\
& =\int_{\Gamma} \frac{\partial u}{\partial n} u \phi^{2}+\int_{\Gamma} \frac{\partial \phi}{\partial n} u^{2} \phi-\frac{1}{2} \int_{\Omega} \nabla u^{2} \nabla \phi^{2}-\int_{\Omega} \Delta \phi u^{2} \phi .
\end{aligned}
$$

Again, integrating by parts in the last integral we have

$$
\begin{aligned}
\int_{\Omega} \Delta \phi u^{2} \phi & =-\int_{\Omega} \nabla \phi \nabla\left(u^{2} \phi\right)+\int_{\Gamma} \frac{\partial \phi}{\partial n} u^{2} \phi \\
& =-\frac{1}{2} \int_{\Omega} \nabla u^{2} \nabla \phi^{2}-\int_{\Omega} u^{2}|\nabla \phi|^{2}+\int_{\Gamma} \frac{\partial \phi}{\partial n} u^{2} \phi .
\end{aligned}
$$

Putting these two inequalities together we obtain the lemma.

Proof of Proposition 3.1. We start by perfoming an analysis in the interior of $\Omega$. For any $\delta>0$ small enough, denote by $U_{\delta}=\{x \in \Omega: d(x, \Gamma) \geq \delta\}$. We are going to show that there exists a constant $C$ independent of $\varepsilon$ and $\delta$ such that

$$
\left\|u_{\varepsilon}-u_{0}\right\|_{L^{\infty}\left(U_{\delta}\right)} \leq C \frac{|\varepsilon \log (\varepsilon)|^{1 / 4}}{\delta^{2}}
$$


Let $\phi_{\delta} \in C_{0}^{\infty}(\Omega)$ a function with the property that $\phi_{\delta}=1$ in $U_{\delta}, \phi_{\delta}=0$ in $\Omega \backslash U_{\delta / 2}=$ $\{x \in \Omega: d(x, \Gamma) \leq \delta / 2\}$ and $\left|\phi_{\delta}(x)\right| \leq 1,\left|\nabla \phi_{\delta}(x)\right| \leq C / \delta$ and $\left|\Delta \phi_{\delta}(x)\right| \leq C / \delta^{2}$. Denote by $z_{\varepsilon}=u_{\varepsilon}-u_{0}$ and by $w=\phi_{\delta} z_{\varepsilon}$. Notice that $w$ depends on both $\delta$ and $\varepsilon$. The function $w$ satisfies the equation:

$$
\left\{\begin{aligned}
\Delta w & =2 \nabla z_{\varepsilon} \nabla \phi_{\delta}+z_{\varepsilon} \Delta \phi_{\delta}, & & \text { in } \Omega, \\
w & =0, & & \text { on } \Gamma .
\end{aligned}\right.
$$

Now we estimate both terms in the right hand side of (3.2).

Applying Lemma 3.2 with $u=z_{\varepsilon}$ and $\phi=\phi_{\delta}$ we obtain that

$$
\int_{U_{\delta}}\left|\nabla z_{\varepsilon}\right|^{2} \leq \int_{\Omega}\left|\nabla\left(z_{\varepsilon} \phi_{\delta}\right)\right|^{2}=\int_{\Omega} z_{\varepsilon}^{2}\left|\nabla \phi_{\delta}\right|^{2} \leq C \frac{|\varepsilon \log (\varepsilon)|^{1 / 2}}{\delta^{2}}, \text { for any } \delta>0,
$$

where we have used (2.3). Appying this estimate to $U_{\delta / 2}$ we obtain

$$
\left\|\nabla z_{\varepsilon} \nabla \phi_{\delta}\right\|_{L^{2}(\Omega)}^{2} \leq C\left\|\nabla z_{\varepsilon}\right\|_{L^{2}\left(U_{\delta / 2}\right)}^{2} \frac{1}{\delta^{2}} \leq C \frac{|\varepsilon \log (\varepsilon)|^{1 / 2}}{(\delta / 2)^{2}} \frac{1}{\delta^{2}} \leq C \frac{|\varepsilon \log (\varepsilon)|^{1 / 2}}{\delta^{4}} .
$$

Similarly, from (2.3).

$$
\left\|z_{\varepsilon} \Delta \phi_{\delta}\right\|_{L^{2}(\Omega)}^{2} \leq C\left\|z_{\varepsilon}\right\|_{L^{2}(\Omega)}^{2} \frac{1}{\delta^{4}} \leq C \frac{|\varepsilon \log (\varepsilon)|^{1 / 2}}{\delta^{4}} .
$$

With these estimates, applying elliptic regularity theory to (3.2) we get

$$
\|w\|_{H^{2}(\Omega)} \leq C \frac{|\varepsilon \log (\varepsilon)|^{1 / 4}}{\delta^{2}} .
$$

Using the embedding $H^{2}(\Omega) \hookrightarrow L^{\infty}(\Omega)$ we obtain

$$
\left\|u_{\varepsilon}-u_{0}\right\|_{L^{\infty}\left(U_{\delta}\right)} \leq\|w\|_{L^{\infty}(\Omega)} \leq C \frac{|\varepsilon \log (\varepsilon)|^{1 / 4}}{\delta^{2}},
$$

where the constant $C$ is independent of $\varepsilon$ and $\delta$. This shows (3.1).

To show the $C_{l o c}^{\infty}(\Omega)$-convergence we proceed as follows. Applying again elliptic regularity theory to equation (3.2) we obtain that for any $k \geq 1$

$$
\left\|u_{\varepsilon}-u_{0}\right\|_{H^{k+1}\left(U_{\delta}\right)} \leq C(\delta)\left\|u_{\varepsilon}-u_{0}\right\|_{H^{k}\left(U_{\delta / 2}\right)} .
$$

Given now any fixed open set $U \subset \Omega$ with $\bar{U} \subset \Omega$, and any $r \geq 1$ we can choose a sequence of $0<\delta_{0}<\delta_{1}<\cdots<\delta_{r}$ with the property that $U_{\delta_{i+1} / 2} \subset U_{\delta_{i}}, i=0, \ldots, r-1$, and $U \subset U_{\delta_{r}}$. 
Using (3.3) we get that

$$
\left\|u_{\varepsilon}-u_{0}\right\|_{H^{i}\left(U_{\delta_{i}}\right)} \leq C\left(\delta_{i}\right)\left\|u_{\varepsilon}-u_{0}\right\|_{H^{i-1}\left(U_{\delta_{i} / 2}\right)} \leq C\left(\delta_{i}\right)\left\|u_{\varepsilon}-u_{0}\right\|_{H^{i-1}\left(U_{\delta_{i-1}}\right)}
$$

which, by repeated iterations, implies that

$$
\left\|u_{\varepsilon}-u_{0}\right\|_{H^{r}(U)} \leq C\left(\delta_{0}, \cdots, \delta_{r}\right)\left\|u_{\varepsilon}-u_{0}\right\|_{L^{2}\left(U_{\delta_{0}}\right)} \leq C(U)|\varepsilon \log (\varepsilon)|^{1 / 4} \rightarrow 0, \quad \text { as } \varepsilon \rightarrow 0 .
$$

Since this is obtained for arbitrary large $r$ we obtain the convergence of the minimizers in $C^{\infty}(U)$. Since $U$ is also arbitrary we get the $C_{l o c}^{\infty}(\Omega)$-convergence. We remark that the constant $C(U)$ depends only on the distance of $U$ to the boundary of $\Omega$.

We analyse now the convergence near $\Gamma$ but outside the transition points. We parameterize $\Gamma$ with a map $\gamma:[0, S] \rightarrow \Gamma$, using for instance the arclength. Observe that by the regularity of $\partial \Omega$ the map $(s, \lambda) \in[0, S] \times(0, \delta) \rightarrow \gamma(s)-\lambda \vec{n} \in \Omega$, where $\vec{n}$ denotes the outward normal direction, is a smooth parameterization of the set $\Omega \backslash U_{\delta}$ as long as $\delta$ is small enough. For any two points $\sigma<\sigma^{\prime}$ and for $\delta$ small enough we denote by

$$
\begin{aligned}
& R_{\sigma, \delta}=\{x=\gamma(\sigma)-\lambda \vec{n}: 0 \leq \lambda \leq \delta\} \\
& K_{\sigma, \sigma^{\prime}, \delta}=\left\{x=\gamma(s)-\lambda \vec{n}, s \in\left[\sigma, \sigma^{\prime}\right] 0 \leq \lambda \leq \delta\right\}=\bigcup_{\sigma \leq s \leq \sigma^{\prime}} R_{s, \delta}, \\
& \Gamma_{\sigma, \sigma^{\prime}}^{0}=\left\{x=\gamma(s), s \in\left[\sigma, \sigma^{\prime}\right]\right\}=\Gamma \cap \partial K_{\sigma, \sigma^{\prime}, \delta} \\
& \Gamma_{\sigma, \sigma^{\prime}, \delta}^{1}=\left\{x=\gamma(s)-\delta \vec{n}, s \in\left[\sigma, \sigma^{\prime}\right]\right\}
\end{aligned}
$$

Notice that with this notations $\partial K_{\sigma, \sigma^{\prime}, \delta}=\Gamma_{\sigma, \sigma^{\prime}}^{0} \cup \Gamma_{\sigma, \sigma^{\prime}, \delta}^{1} \cup R_{\sigma, \delta} \cup R_{\sigma^{\prime}, \delta}$. Denote by $s_{i} \in[0, S]$ the values such that $\gamma\left(s_{i}\right)=x_{i}$ for $i=1, \ldots, 2 N$. Without loss of generality we may assume that $s_{1}=0$. We also denote by $x_{2 N+1}=x_{1}=\gamma(0)=\gamma(S)$, see Figure 1 .

Let us choose in $(3.1) \delta(\varepsilon)=\varepsilon^{\rho}$ for some $\rho<1 / 8$. From $(3.1)$ we still have

$$
\left\|u_{\varepsilon}-u_{0}\right\|_{L^{\infty}\left(U_{\delta(\varepsilon)}\right)} \rightarrow 0, \text { as } \varepsilon \rightarrow 0 .
$$

We are going to show that for any $\sigma<\sigma^{\prime}$ with $\left[\sigma, \sigma^{\prime}\right] \subset\left(s_{i}, s_{i+1}\right)$, for some $i=$ $1, \ldots, 2 N+1$, we have

$$
\left\|u_{\varepsilon}-u_{0}\right\|_{L^{\infty}\left(K_{\sigma, \sigma^{\prime}, \delta(\varepsilon)}\right)} \rightarrow 0, \text { as } \varepsilon \rightarrow 0
$$

Since the function $u_{0}$ is either 1 or 0 in $\Gamma$ and, therefore, it is constant on $\Gamma_{s_{i}, s_{i+1}}^{0}$, $i=1, \ldots, 2 N+1$, we will have that in $\Gamma_{\sigma, \sigma^{\prime}}^{0}, u_{0}$ is either constantly 0 or constantly 1 . Let us assume that $u_{0} \equiv 0$ in $\Gamma_{\sigma, \sigma^{\prime}}^{0}$. The other case is done analogously. Hence, by the continuity of $u_{0}$ outside the transition points and since $\delta(\varepsilon) \rightarrow 0$, we will have that $\left\|u_{0}\right\|_{L^{\infty}\left(K_{\sigma, \sigma^{\prime}, \delta(\varepsilon)}\right)} \rightarrow 0$ as $\varepsilon \rightarrow 0$. Hence, to show (3.5) it will be enough to show

$$
\left\|u_{\varepsilon}\right\|_{L^{\infty}\left(K_{\sigma, \sigma^{\prime}, \delta(\varepsilon)}\right)} \rightarrow 0, \text { as } \varepsilon \rightarrow 0
$$




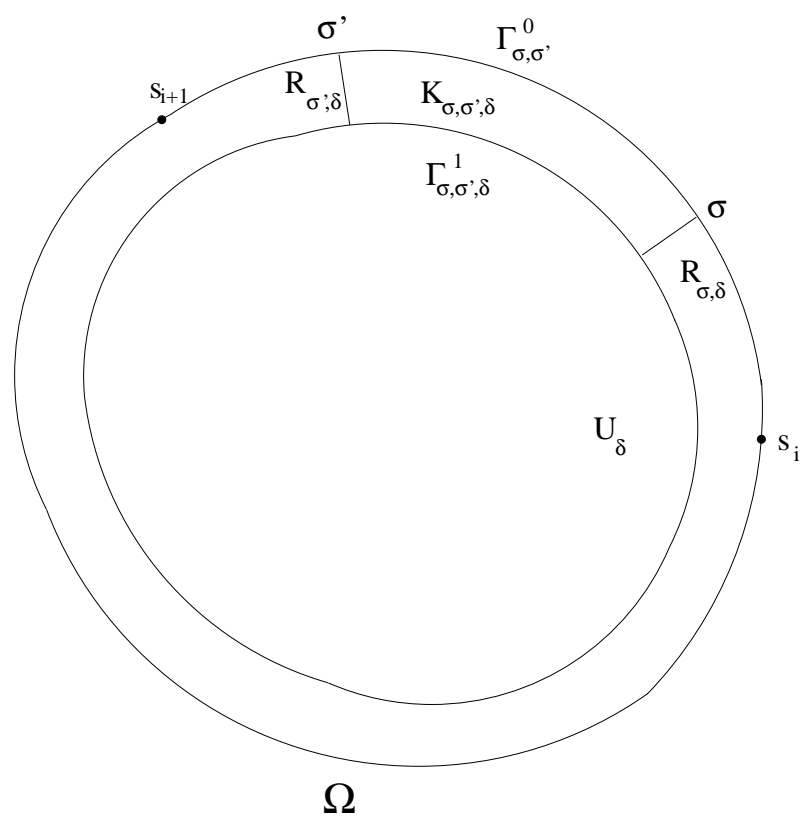

Figure 1:

Let us show now that for any $\theta_{0}$ and $\theta_{1}$ with $\left[\theta_{0}, \theta_{1}\right] \subset\left(s_{i}, s_{i+1}\right)$ we have that

$$
\min _{\theta \in\left[\theta_{0}, \theta_{1}\right]}\left\|u_{\varepsilon}\right\|_{L^{\infty}\left(R_{\theta, \delta(\varepsilon)}\right)} \rightarrow 0, \text { as } \varepsilon \rightarrow 0
$$

Assume this is not true, then there will exist a positive number $\eta>0$ and a sequence of $\varepsilon \rightarrow 0$, that we will still denote by $\varepsilon$, such that for all $\theta \in\left[\theta_{0}, \theta_{1}\right]$ we have $\left\|u_{\varepsilon}\right\|_{L^{\infty}\left(R_{\theta, \delta(\varepsilon)}\right)} \geq \eta$

On the other hand, since we also have $\left\|u_{0}\right\|_{L^{\infty}\left(K_{\theta_{0}, \theta_{1}, \delta(\varepsilon)}\right)} \rightarrow 0$, from (3.4) we obtain that $\left\|u_{\varepsilon}\right\|_{L^{\infty}\left(\Gamma_{\theta_{0}, \theta_{1}, \delta(\varepsilon)}^{1}\right)} \rightarrow 0$. In particular for $\varepsilon$ small enough we will have that $\left\|u_{\varepsilon}\right\|_{L^{\infty}\left(\Gamma_{\theta_{0}, \theta_{1}, \delta(\varepsilon)}^{1}\right)} \leq \eta / 2$.

But then, using the parameterization of $K_{\theta_{0}, \theta_{1}, \delta(\varepsilon)}$ given by $(s, \lambda) \in\left[\theta_{0}, \theta_{1}\right] \times(0, \delta(\varepsilon)) \rightarrow$ $x=\gamma(s)-\lambda \vec{n}$ we have that

$$
\int_{\Omega}\left|\nabla u_{\varepsilon}\right|^{2} d x \geq \int_{K_{\theta_{0}, \theta_{1}, \delta(\varepsilon)}}\left|\nabla u_{\varepsilon}\right|^{2} d x \geq C \int_{\theta_{0}}^{\theta_{1}} \int_{0}^{\delta(\varepsilon)}\left|\frac{\partial u_{\varepsilon}(x(s, \lambda))}{\partial \lambda}\right|^{2} d \lambda d s .
$$

But notice that for $s \in\left[\theta_{0}, \theta_{1}\right]$ fixed, the function $\lambda \rightarrow \psi_{\varepsilon}(\lambda) \equiv u_{\varepsilon}(x(s, \lambda))$ has $\psi(\delta(\varepsilon)) \leq \eta / 2$ and there exists a point $\lambda$ for which $\psi_{\varepsilon}(\lambda) \geq \eta$. This implies that

$$
\int_{0}^{\delta(\varepsilon)}\left|\frac{\partial \psi_{\varepsilon}(\lambda)}{\partial \lambda}\right|^{2} d \lambda=\int_{0}^{\delta(\varepsilon)}\left|\frac{\partial u_{\varepsilon}(x(s, \lambda))}{\partial \lambda}\right|^{2} d \lambda \geq \frac{(\eta / 2)^{2}}{\delta(\varepsilon)}, \quad s \in\left[\theta_{0}, \theta_{1}\right] .
$$


Taking into account that we have chosen $\delta(\varepsilon)=\varepsilon^{\rho}$ for some $\rho<1 / 8$, we obtain that

$$
\int_{\Omega}\left|\nabla u_{\varepsilon}\right|^{2} d x \geq C \frac{(\eta / 2)^{2}}{\delta(\varepsilon)} \geq C \eta^{2} \varepsilon^{-\rho}
$$

which contradicts the fact that $\int_{\Omega}\left|\nabla u_{\varepsilon}\right|^{2} d x \leq C|\log (\varepsilon)|$, as it was shown in (2.2). This shows (3.7).

We are now in position to prove (3.6). For given $\left[\sigma, \sigma^{\prime}\right] \subset\left(s_{i}, s_{i+1}\right)$, choose $\sigma_{0} \in\left(s_{i}, \sigma\right)$ and $\sigma_{0}^{\prime} \in\left(\sigma^{\prime}, s_{i+1}\right)$. Applying $(3.7)$ to $\left[\sigma_{0}, \sigma\right]$ and to $\left[\sigma^{\prime}, \sigma_{0}^{\prime}\right]$ we obtain that

$$
\begin{aligned}
& \min _{\theta \in\left[\sigma_{0}, \sigma\right]}\left\|u_{\varepsilon}\right\|_{L^{\infty}\left(R_{\theta, \delta(\varepsilon)}\right)} \rightarrow 0, \text { as } \varepsilon \rightarrow 0, \\
& \min _{\theta \in\left[\sigma^{\prime}, \sigma_{0}^{\prime}\right]}\left\|u_{\varepsilon}\right\|_{L^{\infty}\left(R_{\theta, \delta(\varepsilon)}\right)} \rightarrow 0, \text { as } \varepsilon \rightarrow 0 .
\end{aligned}
$$

In particular we can choose $\theta(\varepsilon) \in\left[\sigma_{0}, \sigma\right]$ and $\theta^{\prime}(\varepsilon) \in\left[\sigma^{\prime}, \sigma_{0}^{\prime}\right]$ such that

$$
\begin{aligned}
& \left\|u_{\varepsilon}\right\|_{L^{\infty}\left(R_{\theta(\varepsilon), \delta(\varepsilon)}\right)} \rightarrow 0, \text { as } \varepsilon \rightarrow 0, \\
& \left\|u_{\varepsilon}\right\|_{L^{\infty}\left(R_{\theta^{\prime}(\varepsilon), \delta(\varepsilon)}\right)} \rightarrow 0, \text { as } \varepsilon \rightarrow 0 .
\end{aligned}
$$

Moreover, from (3.5) and the fact that $\left\|u_{0}\right\|_{L^{\infty}\left(\Gamma_{\sigma_{0}, \sigma_{0}^{\prime}, \delta(\varepsilon)}^{1}\right)} \rightarrow 0$, as $\varepsilon \rightarrow 0$ we have that

$$
\left\|u_{\varepsilon}\right\|_{L^{\infty}\left(\Gamma_{\theta(\varepsilon), \theta^{\prime}(\varepsilon), \delta(\varepsilon)}^{1}\right)} \rightarrow 0, \text { as } \varepsilon \rightarrow 0
$$

These facts mean that if we consider the set $K_{\theta(\varepsilon), \theta^{\prime}(\varepsilon), \delta(\varepsilon)}$, which contains $K_{\sigma, \sigma^{\prime}, \delta(\varepsilon)}$, in the three parts of the boundary given by $\Gamma_{\theta(\varepsilon), \theta^{\prime}(\varepsilon), \delta(\varepsilon)}^{1}, R_{\theta(\varepsilon), \delta(\varepsilon)}$ and $R_{\theta^{\prime}(\varepsilon), \delta(\varepsilon)}$ the function $u_{\varepsilon}$ goes to 0 in $L^{\infty}$. If it does not go to zero in $L^{\infty}$ in the whole of $K_{\theta(\varepsilon), \theta^{\prime}(\varepsilon), \delta(\varepsilon)}$ we will reach to a contradiction with the fact that $u_{\varepsilon}$ is a minimizer. To see this, consider $\eta \leq \frac{1}{2} \min \left\{G(x, 1): x \in \Gamma_{\sigma_{0}, \sigma_{0}^{\prime}}^{0}\right\}$. Then if we choose $\varepsilon$ small enough such that

$$
\begin{aligned}
& \left\|u_{\varepsilon}\right\|_{L^{\infty}\left(R_{\theta(\varepsilon), \delta(\varepsilon)}\right.} \leq \eta / 2, \\
& \left\|u_{\varepsilon}\right\|_{L^{\infty}\left(R_{\theta^{\prime}(\varepsilon), \delta(\varepsilon)}\right)} \leq \eta / 2, \\
& \left\|u_{\varepsilon}\right\|_{L^{\infty}\left(\Gamma_{\theta(\varepsilon), \theta^{\prime}(\varepsilon), \delta(\varepsilon)}^{1}\right)} \leq \eta / 2
\end{aligned}
$$

and if we define the function

$$
w_{\varepsilon}(x)= \begin{cases}u_{\varepsilon}(x), & \text { outside } K_{\theta(\varepsilon), \theta^{\prime}(\varepsilon), \delta(\varepsilon),}, \\ \min \left\{u_{\varepsilon}(x), \eta\right\}, & K_{\theta(\varepsilon), \theta^{\prime}(\varepsilon), \delta(\varepsilon),}\end{cases}
$$

then, $w_{\varepsilon} \in H^{1}(\Omega)$. If there exists a point in $K_{\theta(\varepsilon), \theta^{\prime}(\varepsilon), \delta(\varepsilon)}$ for which $u_{\varepsilon}>\eta$, then we have that

$$
\int_{\Omega}\left|\nabla w_{\varepsilon}\right|^{2} d x<\int_{\Omega}\left|\nabla u_{\varepsilon}\right|^{2} d x, \quad \text { and } \int_{\Gamma} G\left(x, w_{\varepsilon}(x)\right) d x \leq \int_{\Gamma} G\left(x, u_{\varepsilon}(x)\right) d x
$$


This implies that $J_{\varepsilon}\left(w_{\varepsilon}\right)<J\left(u_{\varepsilon}\right)$, which contradicts the fact that $u_{\varepsilon}$ is a minimizer. This shows that neccesarily

$$
\left\|u_{\varepsilon}\right\|_{L^{\infty}\left(K_{\theta(\varepsilon), \theta^{\prime}(\varepsilon), \delta(\varepsilon)}\right)} \rightarrow 0, \quad \text { as } \varepsilon \rightarrow 0 .
$$

This proves the proposition. $\square$

\section{Stability of profiles}

The patterns $u_{\varepsilon}$ we have found for $\varepsilon$ small enough are near the profile given by the harmonic extension, $u_{0}$, of the characteristic function $\chi$ defined on the boundary. By its definition a pattern is a stable equilibrium of the system and this means that, for $\varepsilon$ fixed, if we start near $u_{\varepsilon}$ then the flow will remain in a neighborhood of the pattern. It is now important to study the stability regions of this equilibria as $\varepsilon \rightarrow 0$. As a matter of fact in this section we will construct a sequence of nested regions $R_{n}$, that is $\cdots \subset R_{n} \subset R_{n-1} \subset \cdots$, around the limiting profile $u_{0}$ with the property that, for $0<\varepsilon<\varepsilon(n)$, if we pick up an initial condition in $R_{n}$ the solution of (1.1) does not leave $R_{n-1}$ for all positive time. Moreover this regions are independent of $\varepsilon$ and their intersection contains only the limiting profile $u_{0}$, see Theorem 4.1. This does not imply that the only equilibrium inside $R_{n}$ is $u_{\varepsilon}$. There may coexist in $R_{n}$ with other equilibria and even some of them may be unstable. But it really ensures that if we start near the limiting profile $u_{0}$ then, for $\varepsilon$ small enough, we will remain close to it for all positive time. In this sense is that we refer to stability of profiles.

The analysis performed so far has been done for the prototype nonlinearity $g(x, u)=$ $u(u-1)(u-c(x))$, but all the results apply for the most general nonlinearity $g_{a, b}(x, u)=$ $(u-a)(u-b)(u-\tilde{c}(x))$ where $a<\tilde{c}(x)<b$ for all $x \in \Gamma$. The role of the constants solutions $u=0, u=1$ is played now by $u=a$ and $u=b$. The transitions will lie now in the points $x_{i}$ that satisfy $\tilde{c}\left(x_{i}\right)=(a+b) / 2$. That is, the points where

$$
\int_{a}^{(a+b) / 2} g_{a, b}\left(x_{i}, u\right) d u+\int_{(a+b) / 2}^{b} g_{a, b}\left(x_{i}, u\right) d u=0 .
$$

Again we will need to assume that $\frac{\partial \tilde{c}}{\partial \tau}\left(x_{i}\right) \neq 0$ at the transition points.

We go back again to our nonlinearity $g(x, u)=u(1-u)(c(x)-u)$ in (1.1), which satisfies that $0<c(x)<1$ on $\Gamma$. Hence there exists $\eta>0$ such that the function $c(x)$ satisfies

$$
\eta<c(x)<1-\eta, \quad \text { for all } x \in \Gamma \text {. }
$$

We consider now the functions

$$
\begin{aligned}
& g_{\delta}^{-}(x, u)=(u+\delta)(1-\delta-u)(c(x)+\delta-u), \\
& g_{\delta}^{+}(x, u)=(u-\delta)(1+\delta-u)(c(x)-\delta-u)
\end{aligned}
$$


for any strictly positive $\delta$. Observe that with the notation above, $g_{\delta}^{-}=g_{-\delta, 1-\delta}$ with $\tilde{c}(x)=c(x)+\delta$ and $g_{\delta}^{+}=g_{\delta, 1+\delta}$ with $\tilde{c}(x)=c(x)-\delta$. Let us remark that the functions $g_{\delta}^{-}(x, \cdot), g(x, \cdot)$ and $g_{\delta}^{+}(x, \cdot)$ are ordered, that is, $g_{\delta}^{+}(x, \cdot)<g(x, \cdot)<g_{\delta}^{-}(x, \cdot)$.

Since the function $c$ is $C^{1}$ and $\frac{\partial c}{\partial \tau}\left(x_{i}\right) \neq 0$, for $\delta$ small enough the functions $g_{\delta}^{-}(x, \cdot)$ and $g_{\delta}^{+}(x, \cdot)$ satisfy the same hypothesis of the function $g(x, \cdot)$. That is, both are cubic functions with the middle zero moving with $x \in \Gamma$ under the given $c(x)$, there exist exactly $2 N$ transition points for each of them, that we denote by $x_{i}^{-}$and $x_{i}^{+}, i=1, \ldots, 2 N$, respectively and the transversality condition is satisfied for the transition points of each of them. Let us note that the transition points satisfy now $c\left(x_{i}^{-}\right)=1 / 2-2 \delta$ for $g_{\delta}^{-}(x, \cdot)$ and $c\left(x_{i}^{+}\right)=1 / 2+2 \delta$ for $g_{\delta}^{+}(x, \cdot), i=1, \ldots, 2 N$.

Let us observe that, we have $x_{i}^{-}<x_{i}<x_{i}^{+}$if $\frac{\partial c}{\partial \tau}\left(x_{i}\right)>0$ or $x_{i}^{+}<x_{i}<x_{i}^{-}$if $\frac{\partial c}{\partial \tau}\left(x_{i}\right)<0$

Now, we take the problem (1.1) for $g_{\delta}^{-}(x, v)$, that is,

$$
\left\{\begin{array}{rlrl}
v_{t}-\varepsilon \Delta v & =0, & & \text { in } \Omega, t>0 \\
\varepsilon \frac{\partial v}{\partial n}+g_{\delta}^{-}(x, v) & =0, & & \text { on } \Gamma, t>0 \\
v(\cdot, 0) & =\psi^{-}(\cdot) \in H^{1}(\Omega), &
\end{array}\right.
$$

and for $g_{\delta}^{+}(x, v)$, that is,

$$
\left\{\begin{array}{rlrl}
v_{t}-\varepsilon \Delta v & =0, & & \text { in } \Omega, t>0 \\
\varepsilon \frac{\partial v}{\partial n}+g_{\delta}^{+}(x, v) & =0, & & \text { on } \Gamma, t>0 \\
v(\cdot, 0) & =\psi^{+}(\cdot) \in H^{1}(\Omega) . &
\end{array}\right.
$$

We consider as well the energy functionals $J_{\varepsilon, \delta}^{-}, J_{\varepsilon, \delta}^{+}: H^{1}(\Omega) \rightarrow \mathbb{R}$, defined as in the first section:

$$
\begin{aligned}
& J_{\varepsilon, \delta}^{-}(v)=\frac{\varepsilon}{2} \int_{\Omega}|\nabla v|^{2}+\int_{\Gamma} G_{\delta}^{-}(x, v) d x, \\
& J_{\varepsilon, \delta}^{+}(v)=\frac{\varepsilon}{2} \int_{\Omega}|\nabla v|^{2}+\int_{\Gamma} G_{\delta}^{+}(x, v) d x,
\end{aligned}
$$

where $G_{\delta}^{-}(x, v)=\int_{0}^{v} g_{\delta}^{-}(x, s) d s$ and $G_{\delta}^{+}(x, v)=\int_{0}^{v} g_{\delta}^{+}(x, s) d s$.

For a given $\varepsilon>0$, let $u_{\varepsilon, \delta}^{-}$and $u_{\varepsilon, \delta}^{+}$be absolute minimizers of $J_{\varepsilon, \delta}^{-}$and $J_{\varepsilon, \delta}^{+}$, respectively. Finally, let us denote by $\chi_{\delta}^{-}$and $\chi_{\delta}^{+}$the characteristic functions corresponding to the extreme zeros of $g_{\delta}^{-}(x, u)$ and $g_{\delta}^{+}(x, u)$, defined in the same way as the function $\chi$ with respect to $g(x, u)$, in Section 1. Let $u_{0, \delta}^{-}$and $u_{0, \delta}^{+}$be the harmonic extensions to $\Omega$ of the functions $\chi_{\delta}^{-}$and $\chi_{\delta}^{+}$both defined on $\Gamma$, respectively. By the construction we obviously 
have that $\chi_{\delta}^{-}<\chi<\chi_{\delta}^{+}$, which implies by the maximum principle that $u_{0, \delta}^{-}<u_{0}<u_{0, \delta}^{+}$. Similarly, if we have $\delta<\delta^{\prime}$ we also obtain the orderings $\chi_{\delta^{\prime}}^{-}<\chi_{\delta}^{-}<\chi<\chi_{\delta}^{+}<\chi_{\delta^{\prime}}^{+}$, and again by the maximum principles we get the same ordering relations for the harmonic extensions, that is $u_{0, \delta^{\prime}}^{-}<u_{0, \delta}^{-}<u_{0}<u_{0, \delta}^{+}<u_{0, \delta^{\prime}}^{+}$, see Figure 2.

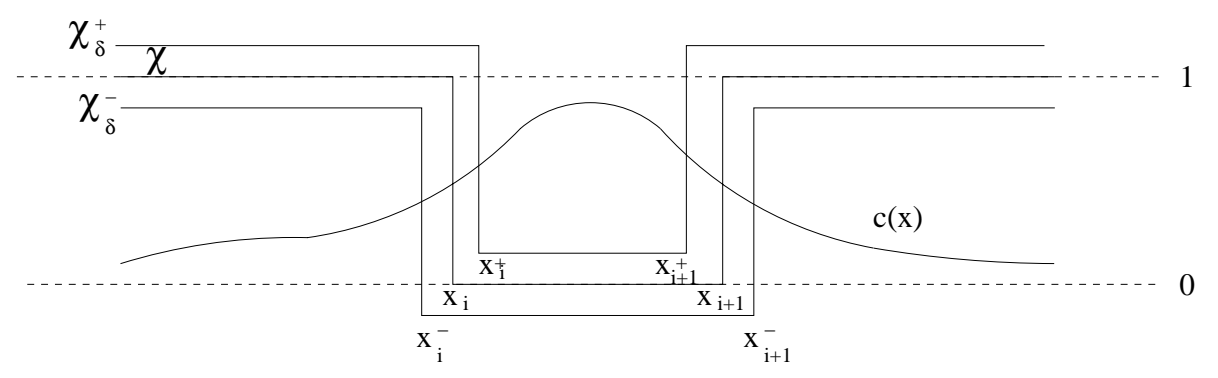

Figure 2:

The main result in this section is the next theorem.

Theorem 4.1 Given any $\delta>0$ small, there exists $\varepsilon(\delta)>0$ such that for any $\omega_{\varepsilon} \in$ $\left[u_{0, \delta}^{-}, u_{0, \delta}^{+}\right]$the solution of (1.1) with initial condition $\omega_{\varepsilon}$, that we will denote by $u_{\varepsilon}\left(t, \omega_{\varepsilon}\right)$, satisfies $u_{\varepsilon}\left(t, \omega_{\varepsilon}\right) \in\left[u_{0,2 \delta}^{-}, u_{0,2 \delta}^{+}\right]$, for all $0<\varepsilon<\varepsilon(\delta), t \geq 0$.

Remark 4.2 Observe that the ordered intervals $\left[u_{0, \delta}^{-}, u_{0, \delta}^{+}\right]$are nested and that $\bigcap_{\delta>0}\left[u_{0, \delta}^{-}, u_{0, \delta}^{+}\right]=$ $\left\{u_{0}\right\}$. In particular Theorem 4.1 implies a stability of the profile given by $u_{0}$, in the sense that if we start with a profile near $u_{0}$, then for $\varepsilon$ small enough, we will remain close to it.

Before proving this result we construct, for a given $\varepsilon>0$, a region which is positively invariant for the flow defined by (1.1), that is, a region with the property that if an initial condition is taken in this region the solution of (1.1) does not leave the region for any $t>0$.

Lemma 4.3 Given any $\delta>0$ small enough, the functions $u_{\varepsilon, \delta}^{+}, u_{\varepsilon, \delta}^{-}$are a super and subsolution of the problem (1.3), respectively. Moreover, there exists $\varepsilon(\delta)>0$ such that $u_{\varepsilon, \delta}^{-}<u_{\varepsilon}<u_{\varepsilon, \delta}^{+}$for all $0<\varepsilon<\varepsilon(\delta)$.

We remind that $u^{+}$is a supersolution of (1.3) if it satisfies

$$
\left\{\begin{aligned}
-\varepsilon \Delta u^{+} \geq 0, & & \text { in } \Omega, \\
\varepsilon \frac{\partial u^{+}}{\partial n}+g\left(x, u^{+}\right) \geq 0, & & \text { on } \Gamma .
\end{aligned}\right.
$$


If $u^{-}$satisfies the reverse inequalities, $u^{-}$is a subsolution of (1.3).

Proof. Since $g_{\delta}^{+}(x, u)<g(x, u)<g_{\delta}^{-}(x, u)$ for all $x \in \Gamma$ and $u_{\varepsilon, \delta}^{-}, u_{\varepsilon, \delta}^{+}$are solutions of (4.1) and (4.2), respectively, we conclude that they are sub and supersolutions, respectively, of $(1.3)$.

Now, we are going to see for a fixed and small $\delta$ and for all $\varepsilon$ small enough $u_{\varepsilon, \delta}^{-}, u_{\varepsilon}$ and $u_{\varepsilon, \delta}^{+}$are ordered, for all $x \in \bar{\Omega}$. Applying Proposition 3.1, which states the $L^{\infty}$ convergence far from the transition points of the minimizers to the appropriate characteristic functions, we have that $u_{\varepsilon, \delta}^{-}$goes to $\chi_{\delta}^{-}$and $u_{\varepsilon, \delta}^{+}$goes to $\chi_{\delta}^{+}$, as $\varepsilon \rightarrow 0$, far from their respective transition points. Moreover, by the maximum principles we have the apriori bounds $-\delta<u_{\varepsilon, \delta}^{-}<1-\delta$ and $\delta<u_{\varepsilon, \delta}^{+}<1+\delta$.

Using these two facts and since $\chi_{\delta}^{-}<\chi<\chi_{\delta}^{+}$we conclude the proof of the lemma.

Considering now the evolution problem (1.1), the existence of these $u_{\varepsilon, \delta}^{+}, u_{\varepsilon, \delta}^{-}$and the monotonicity properties of (1.1) we have the next result.

Corollary 4.4 For a given $\delta>0$ small, and for all $\varepsilon>0$ small enough the region $\left[u_{\varepsilon, \delta}^{-}, u_{\varepsilon, \delta}^{+}\right]$is positive invariant under the flow defined by (1.1).

Moreover we have the stability of profiles that we assert in the previous Theorem 4.1 in some regions $\varepsilon$-independent, as we are going to see in the next proof.

Proof of Theorem 4.1 For a given $\delta$ we have that

$$
u_{0,2 \delta}^{-}<u_{0,3 \delta / 2}^{-}<u_{0, \delta}^{-}<u_{0}<u_{0, \delta}^{+}<u_{0,3 \delta / 2}^{+}<u_{0,2 \delta}^{+}
$$

From the $L^{\infty}$ convergence of minimizers outside the transition points we have that there exists $\varepsilon(\delta)$ small enough such that, for any $0<\varepsilon<\varepsilon(\delta)$,

$$
\left[u_{0, \delta}^{-}, u_{0, \delta}^{+}\right] \subset\left[u_{\varepsilon, 3 \delta / 2}^{-}, u_{\varepsilon, 3 \delta / 2}^{+}\right] \subset\left[u_{0,2 \delta}^{-}, u_{0,2 \delta}^{+}\right] .
$$

Since by Corollary 4.4 we have that $\left[u_{\varepsilon, 3 \delta / 2}^{-}, u_{\varepsilon, 3 \delta / 2}^{+}\right]$is invariant, we obtain that for any function $w_{\varepsilon} \in\left[u_{0, \delta}^{-}, u_{0, \delta}^{+}\right]$its evolution remains in $\left[u_{\varepsilon, 3 \delta / 2}^{-}, u_{\varepsilon, 3 \delta / 2}^{+}\right]$and therefore it can not leave the region $\left[u_{0,2 \delta}^{-}, u_{0,2 \delta}^{+}\right] \cdot \square$

Remark 4.5 The theorem above shows that if we start with an initial condition $w_{\varepsilon} \in$ $\left[u_{0, \delta}^{-}, u_{0, \delta}^{+}\right]$its evolution does not leave the region $\left[u_{0,2 \delta}^{-}, u_{0,2 \delta}^{+}\right]$. As a matter of fact, using maximum principles it is easy to see that its $\omega$-limit set for $T_{\varepsilon}(t), 0<\varepsilon<\varepsilon(\delta)$, needs to lie in the interval $\left[u_{0,2 \delta}^{-}, u_{0,2 \delta}^{+}\right] \cap[0,1]$. 


\section{References}

[1] S. Angenent, J. Mallet-Paret, L. Peletier, "Stable Transition Layers in a Semilinear Boundary Value Problem", Journal of Differential Equations 67, 212-242 (1987).

[2] J.M. Arrieta, A.N. Carvalho and A. Rodríguez-Bernal, "Parabolic Problems with Nonlinear Boundary Conditions and Critical Nonlinearities". Journal of Differential Equations 156, 376-406 (1999).

[3] J.M. Arrieta, A.N. Carvalho and A. Rodríguez-Bernal, "Attractors of Parabolic Problems with Nonlinear Boundary Conditions. Uniform Bounds", Comm. in Partial Diff. Equations 25, 1-37 (2000).

[4] J.M. Arrieta, N. Cónsul, A. Rodríguez-Bernal, "Pattern Formation from Boundary Reaction, Fields Inst. Comm. 31, (2002).

[5] N. Cónsul, "On Equilibrium Solutions of Diffusion Equations with Nonlinear Boundary Conditions", Z. Angew. Math. Phys. 47, 194-209 (1996).

[6] N. Cónsul, J. Solà-Morales, "Stable Nonconstant Equilibria in Parabolic Equations with Nonlinear Boundary Conditions", C.R. Acad. Sci. Paris, T. 321, Sèrie I, 299-304 (1995).

[7] N. Cónsul, J. Solà-Morales, "Stability of Local Minima and Stable Nonconstant Equilibria", Journal of Differential Equations 157, 61-81 (1999).

[8] A. do Nascimento, "Inner Transition Layers in a Elliptic Boundary Value Problem", Nonl. Anal.: Th. Meth. and Appl., (to appear).

[9] R. Casten, C. Holland, "Stability properties of solutions to systems of reactiondiffusion equations", SIAM J. Appl. Math. 33, (1977), 353-364

[10] H. Matano, "Asymptotic behavior and stability of solutions of semilinear diffusion equations", Publ. Res. Inst. Math. Sci. 15 (1979), 401-451.

[11] C. Rocha, "Examples of Attractors in Scalar Reaction Diffusion Equations", Journal of Differential Equations 73, 178-195 (1988).

José M. Arrieta

Departamento de Matemática Aplicada

Universidad Complutense de Madrid

28040 Madrid, Spain.

arrieta@sunma4.mat.ucm.es

Neus Cónsul

Departament de Matemàtica Aplicada I 
Universitat Politècnica de Catalunya 08028 Barcelona, Spain

neus@ma1.upc.es

Anibal Rodríguez-Bernal

Departamento de Matemática Aplicada

Universidad Complutense de Madrid

28040 Madrid, Spain

arober@sunma4.mat.ucm.es 